\title{
Spin diffusion in one-dimensional classical Heisenberg model
}

\author{
Debarshee Bagch $*$ \\ Theoretical Condensed Matter Physics Division, \\ Saha Institute of Nuclear Physics, \\ 1/AF Bidhan Nagar, Kolkata 700064, India.
}

(Dated: July 24, 2018)

\begin{abstract}
The problem of spin diffusion is studied numerically in one-dimensional classical Heisenberg model using a deterministic odd even spin precession dynamics. We demonstrate that spin diffusion in this model, like energy diffusion, is normal and one obtains a long time diffusive tail in the decay of autocorrelation function (ACF). Some variations of the model with different coupling schemes and with anisotropy are also studied and we find normal diffusion in all of them. A systematic finite size analysis of the Heisenberg model also suggests diffusive spreading of fluctuation, contrary to previous claims of anomalous diffusion.
\end{abstract}

The classical Heisenberg model [1, 2] has been extensively studied, both analytically and numerically, for several decades and has become a prototypical model for magnetic insulators. However, one important question that still awaits a conclusive answer is regarding the time dependent behavior of the spins, particularly at very high temperature. In the hydrodynamic limit, the dominant mode of fluctuation spreading in this system is believed to obey of the standard diffusion phenomenology. In absence of any microscopic theoretical formalism, studies were mostly numerical, generally involving calculation of time correlation functions. Although the phenomenology of spin diffusion is an old concept [3, 4], its validity in classical Heisenberg model has been vigourously debated in recent times. Although much effort 13 20 has been devoted to understand whether spin diffusion in this system is normal or anomalous, a convincing conclusion is yet to be reached. Settling this question is not only conceptually important e.g., in understanding transport properties of spin systems, but also has direct implications in routinely performed experiments e.g., NMR and ESR in magnetic compounds [5 9]. In the following, we present a brief outline of the diffusion phenomenology and review some of the earlier studies in this direction.

Let us consider a one-dimensional chain containing Heisenberg spins $\left\{\vec{S}_{i}\right\}$ (three dimensional unit vectors) where, $i=1,2, \ldots, N$ with periodic boundary conditions, i.e., $\vec{S}_{N+1} \equiv \vec{S}_{1}$. The Hamiltonian is given by,

$$
\mathcal{H}=-\sum_{i=1}^{N} K_{i} \vec{S}_{i} \cdot \vec{S}_{i+1},
$$

where $K_{i}$ is the interaction strength between the spins $\vec{S}_{i}$ and $\vec{S}_{i+1}$; the spin-spin coupling is ferromagnetic for $K_{i}>0$ and anti-ferromagnetic if $K_{i}<0$. The microscopic equation of motion can be written as,

$$
\frac{d}{d t} \vec{S}_{i}=\vec{S}_{i} \times \vec{B}_{i}
$$

where $\vec{B}_{i}=K_{i-1} \vec{S}_{i-1}+K_{i} \vec{S}_{i+1}$ is the local molecular field experienced by the spin at site $i$. Clearly, Eq. (2) conserves (i) the total energy $E=\sum_{i} E_{i}=-\sum_{i} K_{i} \vec{S}_{i}$. $\vec{S}_{i+1}$, and (ii) the total spin $\vec{S}=\sum_{i} \vec{S}_{i}$.

Since there is no long range order in this system at any finite temperature and because of the conservation of total spin, the spin fluctuation in the hydrodynamic limit is expected to follow a continuity (diffusion) equation $\partial_{t} \vec{S}_{q}(t)=-D_{s} q^{2} \vec{S}_{q}(t)$, where $\vec{S}_{q}(t)$ is the (discrete) Fourier transform of $S_{i}(t)$ and $D_{s}$ is the spin diffusion constant. A similar equation holds for the energy density (since total energy is also a constant of motion). The continuity equation implies that in the hydrodynamic limit (small $q$ and large $t$ ) the spin-spin correlation function $A_{s}(\vec{q}, t) \equiv\left\langle\vec{S}_{q}(t) \cdot \vec{S}_{-q}(0)\right\rangle$ decays with time exponentially, i.e., $A_{s}(\vec{q}, t) \sim e^{-D_{s} q^{2} t}$. A direct consequence of this is that the spin autocorrelation function (ACF) $A_{s}(t) \equiv \frac{1}{N} \sum_{i}\left\langle\vec{S}_{i}(t) \cdot \vec{S}_{i}(0)\right\rangle$ at late times decays with a power law tail $A_{s}(t) \sim t^{-\alpha}$. As predicted by the diffusion phenomenology, the exponent $\alpha$ is equal to $1 / 2$ in one dimension. This is also true for energy ACF $A_{e}(t) \equiv \frac{1}{N} \sum_{i}\left\langle E_{i}(t) E_{i}(0)\right\rangle \sim t^{-1 / 2}$.

Recently, the problem of spin diffusion in this model was studied by Müller [13] and it was reported that $\alpha=0.609 \pm 0.005$ (1D) in the hydrodynamic limit, thus significantly differing from the spin diffusion prediction. Following this, Gerling et. al. [14] performed extensive numerical studies with larger system sizes and for longer times. They strongly opposed the claim made in Ref. [13] and demonstrated that the slope of the ACF slowly decreases as $t$ is increased. Nevertheless, they suggested that the problem is computationally difficult since the non-asymptotic behaviour of the spin $\mathrm{ACF}$ is quite pronounced. In yet another work 15, it was concluded that in numerical simulation it is not possible to observe the $t^{-1 / 2}$ behavior, even if it exists, due to the fact that the numerical scheme introduces computational errors and this violated the conservation of total spin $\vec{S}$. The error propagation affects the decay of the ACF and makes it anomalous. It was suggested that the correlation function may show a crossover from non-diffusive to diffusive behavior and the characteristic crossover time will de- 
pend on the precision of the numerical scheme employed.

Another subsequent numerical work in this direction [16] however claimed that although energy diffusion is normal, spin diffusion has an anomalous behavior. 17]. A coupled-mode theory of spin fluctuation [18] suggested that spin diffusion is anomalous with $A_{s}(t) \sim t^{-2 / 5}$ asymptotically. Authors in Ref. [19] studied few variants of the Heisenberg models (alternate coupling, random coupling etc.) and suggested that spin diffusion is probably normal with alternate coupling but is anomalous with random coupling. A nonlinear dynamics study [20] presented numerical results in support of anomalous diffusion and computed $z \approx 1.67$, which implies $\alpha=z^{-1} \approx 0.6$. Thus, most of the previous works refute the validity of normal spin diffusion in this system.

In this work, we re-investigate spin diffusion in classical Heisenberg model on a ring, using a discrete time odd even dynamics (DTOE) [11]. We compute the temporal decay of the energy and spin ACFs, and its cumulative average (defined later). We perform extensive simulation on large system sizes $(N=5000-20000)$ and for very large times $\left(t=10^{6}\right)$. To the best of our knowledge, such large scale simulation has never been performed in this system to study spin diffusion. We demonstrate convincingly that spin diffusion is normal and the exponent $\alpha=1 / 2$ (within numerical accuracy). We also study a few other variants of the usual Heisenberg model and show that diffusion process is also normal in those cases. For small system sizes, the ACFs saturate after a characteristic timescale that depends on the system size. We have performed systematic finite size analysis for small systems which again indicates that spin diffusion is indeed normal. In the following, we describe our numerical scheme and present the results.

a. DTOE dynamics: This method of integrating the discretised version of equation of motion has been discussed in detail elsewhere (see Ref. [11]). For the sake of completeness, we present an outline of the DTOE dynamics here.

To integrate the equation of motion numerically, one would naively consider a finite difference equation of the Euler form

$$
\vec{S}_{i, t+1}=\vec{S}_{i, t}+\Delta t[\vec{S} \times \vec{B}]_{i, t}
$$

and update all the spins at time $t$ and obtain their values at the next time-step $t+1$. However, it can be shown that using Eq. (3) directly and updating all the spins simultaneously, lead to the violation of the conservation laws stated above for all $\Delta t>0$; the length of the spins is also not held constant [11]. A way to naturally preserve the length of spins $\left|\vec{S}_{i}\right|$ is by using an alternative spin precession update equation (instead of Eq. 3)

$\vec{S}_{i, t+1}=[\vec{S} \cos \phi+(\vec{S} \times \hat{B}) \sin \phi+(\vec{S} \cdot \hat{B}) \hat{B}(1-\cos \phi)]_{i, t}$,

where $\hat{B}_{i}=\vec{B}_{i} /\left|\vec{B}_{i}\right|$ and $\phi_{i}=\left|\vec{B}_{i}\right| \Delta t[12]$. This will, however, still violate the conservation of total energy and total spin.

For the conservation of total energy, we use an oddeven spin update rule where the dynamics described in Eq. (4) is numerically implemented by alternate parallel updates of the spins on odd and even sublattices. Thus, at each step, first, only even spins are updated using the spin precession dynamics Eq. (44) while the odd spins are kept unaltered. Next, the spins on the odd sublattice are similarly updated. It is straight forward to check that update of any spin $\vec{S}_{i}$ affects only the energy of the neighbouring bonds $\epsilon_{i-1}$ and $\epsilon_{i}$, but their sum $\left(\epsilon_{i-1}+\epsilon_{i}\right)$ remains constant. Thus DTOE dynamics conserves energy strictly and also naturally maintains the individual spin lengths.

However, the total spin $\vec{S}$ does not remain conserved using DTOE dynamics. This is a general problem with any standard integration scheme; the conservations are only approximately maintained depending on the accuracy of the scheme.

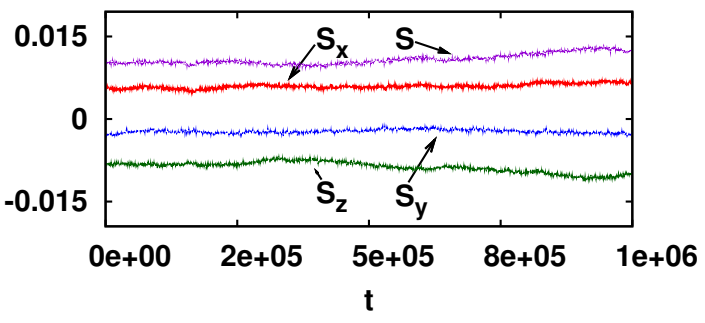

FIG. 1. (Color online) Typical evolution of the magnitude of the total spin $S \equiv|\vec{S}|$ and its components $S_{x}, S_{y}, S_{z}$ using DTOE dynamics with $N=5000, \Delta t=0.05$ and with uniform coupling $K_{i}=1$. All the four quantities are scaled by a factor of $1 / N$.

We will show that DTOE dynamics is still a better numerical scheme compared to other conventional schemes (e.g. Euler method, Runge-Kutta method) since the accuracy here is naturally higher for any given value of $\Delta t$ (as $E$ and $\left|\vec{S}_{i}\right|$ remain accurately conserved for any arbitrary $\Delta t$ ). Thus, one can choose a relatively larger $\Delta t$ without accumulating large numerical errors and thus probe the time dependent behavior of the system at very late times. Although choosing a larger $\Delta t$ essentially converts the equation of motion to a map, it can be shown that independent of the value of $\Delta t$, this dynamics allows the system to settle to the correct equilibrium state 11] and one obtains the correct static spin correlations. Also, we have verified it thoroughly that the asymptotic behavior of the ACFs remains unaltered with smaller $\Delta t$, only the computation becomes more time consuming.

The time evolution of the total spin and its components, from typical run using the DTOE dynamics, is shown in Fig. 1. We find that up to large times $\left(t \sim 10^{6}\right)$, the total spin conservation is approximately maintained; the magnitude $S$ and the components $S_{x}, S_{y}, S_{z}$ do not show any trend of an overall growth (or decay) with time. 
An Euler-like scheme for the same values of the parameters $N$ and $\Delta t$ will however develop numerical instabilities and 'blow up' much before $t \sim 10^{6}$. Thus, using DTOE dynamics, one can perform extensive numerical simulation of the system and reliably determine the behavior of the system in the hydrodynamic limit. Below, we mention the details of our numerical simulation and present the results.

b. Results: First, we simulate the case for which all interactions are positive (ferromagnetic) and uniform i.e., $K_{i}=K$ (and set to unity without the loss of generality). Starting from a random initial spin configuration, we evolve the system using DTOE dynamics. We compute the ACFs $A_{e}(t)$ and $A_{s}(t)$, and study their late time power law decay. However, the ACF has a slow convergence to its asymptotic behavior. Again, in course of its decay with time, the ACF either saturates if the system size is small or, if the system size is large enough, the ACF continues to decay and its the numerical value keeps decreasing. As such, it becomes computationally more and more expensive to get well averaged data at late times. To circumvent these problems, instead of the the ACF, we compute its cumulative average, defined as

$$
C_{s}(t)=\frac{1}{t} \int_{0}^{t} A_{s}(\tau) d \tau
$$

where $A_{s}(t)=\frac{1}{N} \sum_{i=1}^{N}\left\langle\vec{S}_{i}(0) \cdot \vec{S}_{i}(t)\right\rangle$. This cumulative autocorrelation function $(\mathrm{CACF}) C_{s}(t)$ has the same asymptotic time dependence as the ACF and therefore at late times $C_{s}(t) \sim t^{-1 / 2}$, if spin diffusion is normal. Moreover, this has the added advantage that the data for $C_{s}(t)$ is much less noisy than that of $A_{s}(t)$ and therefore its asymptotic time dependence can be computed with high accuracy. Likewise, one can define a cumulative average $C_{e}(t)$ for $A_{e}(t)$.

The functions $C_{e}(t)$ and $C_{s}(t)$ obtained using DTOE dynamics are shown in Fig. 2. At late times $\left(\sim t>10^{4}\right)$, we find that all the curves show a clear convergence to $t^{-1 / 2}$ (broken lines in the figures). The $t^{-1 / 2}$ decay of the energy CACF (Fig. 2a) does not come as a surprise since energy diffusion in this model was already known to be normal. It has also been recently shown that energy transport in this model obeys Fourier's law 10, 11] for any nonzero temperature. However, the decay of the spin CACF (Fig. 2b) with an exponent $\alpha=1 / 2$ for almost two decades $\left(\sim 10^{4}-10^{6}\right)$ is quite interesting. In fact, this clearly indicates that, contrary to previous claims, spin diffusion is normal in the classical Heisenberg spin system and spin fluctuation spreads diffusively.

We have also simulated this model with other coupling schemes, namely, with alternate coupling $K_{i}=(-1)^{i}$, and random coupling ( $K_{i}= \pm 1$ assigned randomly) that have been studied in Ref. [19]. The authors of Ref. [19] suggested that spin diffusion appears to be diffusive for the alternate coupling case, whereas, for random coupling, it is probably non-diffusive. However, for both the cases we find a clear $t^{-1 / 2}$ behavior at late times. This is
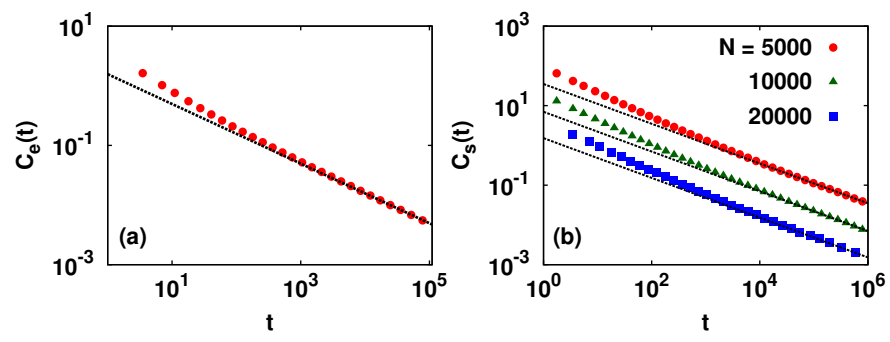

FIG. 2. (Color online) Log-log plot of the autocorrelation functions for the uniform case (a) $C_{e}(t)$ for $N, \Delta t=20000,0.1$ averaged over 100 independent realisations. (b) $C_{s}(t)$ for $N, \Delta t=(5000,0.05),(10000,0.05)$ and $(20000,0.1)$, and averaged over 200,100 , and 25 independent realisations respectively. The data points are shifted along the $y$-axis for better visibility. The broken lines have a slope -0.5
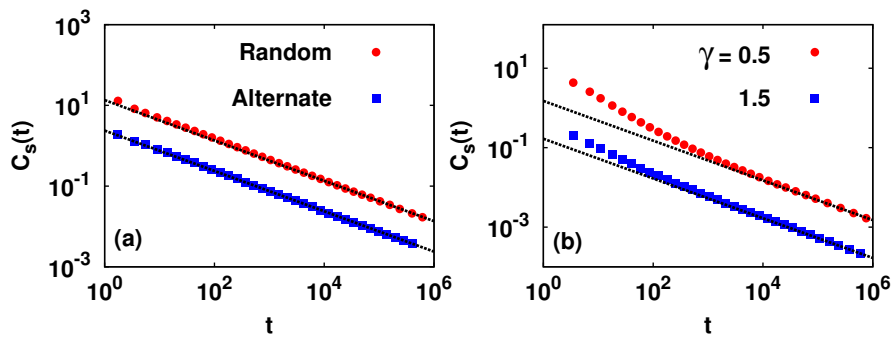

FIG. 3. (Color online) Log-log plot of the autocorrelation function $C_{s}(t)$ (a) for random coupling (circles) and alternate coupling (squares) for $N=20000$ with $\Delta t=0.05$ (b) with anisotropy $\gamma=0.5$ (circles), 1.5 (squares) for $N=20000$ with $\Delta t=0.10$. The data is averaged over 100 independent realisations and shifted along $y$-axis for clarity. The broken lines have a slope -0.5 .

shown in Fig. [3a. Thus, our numerical results convincingly demonstrate that in all the three models namely, uniform, alternate and random coupling, the diffusion process is not anomalous.

We have studied spin diffusion in classical Heisenberg model with anisotropic coupling in different spin directions

$$
\mathcal{H}_{X X Z}=-K \sum_{i=1}^{N}\left[S_{i}^{x} S_{i+1}^{x}+S_{i}^{y} S_{i+1}^{y}+\gamma S_{i}^{z} S_{i+1}^{z}\right],
$$

where $\gamma$ is the anisotropy parameter. Spin diffusion with anisotropy in the classical limit has been studied in some detail recently 21 23. Our data for $C_{s}(t)$ with $\gamma>1$ and $\gamma<1$ is shown in Fig. 3 b. The data shows that at late times $C_{s}(t) \sim t^{-1 / 2}$ and thus indicates normal spin diffusion in the anisotropic model also.

Using finite size analysis of the ACF, one can have an alternative method of estimating the exponent $\alpha$ [20]. We work with small system sizes $N \leq 200$ Heisenberg spins on a ring with uniform coupling $K_{i}=1$. As stated earlier, $A_{s}(t)$ for small system size saturates at some characteristic relaxation time $t_{s}(N)$. The saturation of ACF for different $N$ is shown in Fig. 保. Numerically, we es- 

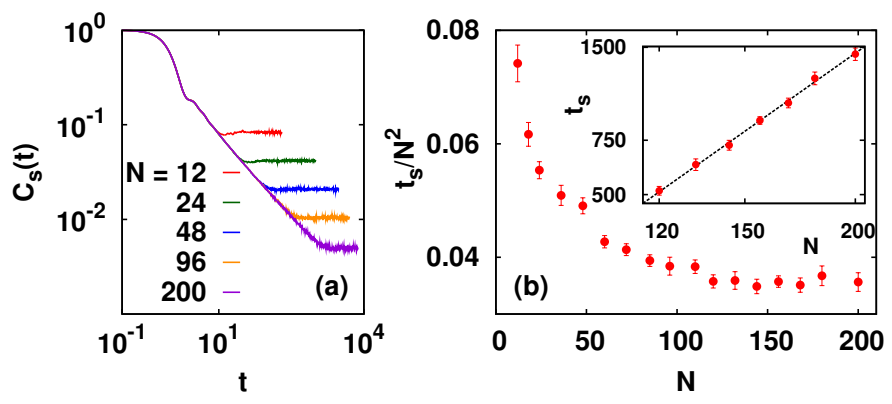

FIG. 4. (Color online) (a) $A_{s}(t)$ for finite systems $N$ saturate at different characteristic times $t_{s}(N)$. (b) Plot of $t_{s}(N) / N^{2}$ as a function of $N$ which roughly becomes constant for $N>$ 100. The inset shows a log-log plot of $t_{s}(N)$ vs. $N$; the best fitted straight line for $N \geq 120$ gives a slope $z=2.02 \pm 0.04$.

timate $t_{s}(N)$ by the intersection of a power law fit, for the linear part of the curve (in logarithmic scale) before it saturates, and the straight line $y=A_{s}(\infty)$, where $A_{s}(\infty)$ is the saturation value of $A_{s}(t)$ at late times. The saturation $A_{s}(\infty)$ is numerically computed by averaging $A_{s}(t)$ far away from the saturation point, i.e., for $t \gg t_{s}$. The relaxation timescale $t_{s}(N)$ is related to the system size $N$ via the dynamical exponent $z$ as $t_{s} \sim N^{z}$. For a diffusive process $z$ should be equal to 2 , which is indeed the case

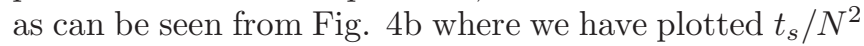
against $N$; the data attains a constancy (approximately) for $N>100$. We fit the data for $N>100$ which is shown in the inset of Fig. 4b. The best fitted straight line in the log-log plot has a slope $z=2.02 \pm 0.04$. Hence, we have $\alpha=z^{-1} \approx 0.5$, implying normal spin diffusion. The value $z=1.67$ reported earlier [20] seems to be due to the smaller system sizes studied there.

To summarize, we have revisited the spin diffusion problem in classical Heisenberg spin model in one dimension. We have performed extensive simulation of the model using DTOE dynamics that preserves the conservations of the total energy $E$ accurately. Although this dynamics is identical to the equation of the motion only in the $\Delta t \rightarrow 0$ limit, it equilibrates the system to the correct stationary state for any finite $\Delta t$ [1]. It is thus advantageous here to use a relatively larger $\Delta t$ and probe the dynamical behavior of the system up to late times. By computing the autocorrelation functions we show that, similar to energy diffusion, spin diffusion in classical one dimensional Heisenberg model is normal $(\alpha=1 / 2)$, contrary to what has been suggested in some of the previous works. We obtain an estimate for the dynamical exponent $z \approx 2$, which again indicates that spin diffusion is normal. The probable reasons as to why most of the previous works concluded that diffusion in this system is anomalous could be because of (a) small scale simulations - both in system size and time, (b) the noisy correlation function data, and (c) the accuracy of the method used. Our way of simulation and analysis take care of most of these issues and produce a clear long time diffusive tails for the correlation function. Although this dynamics still lacks the strict total spin conservation, however unlike conventional integration schemes where the errors accumulation is relatively fast, here the total spin conservation is approximately preserved allowing one to measure the autocorrelation functions up to very large time. It remains a challenge to find a suitable dynamics for this model, which will strictly preserves both energy and spin conservation.

Acknowledgement: The author would like to thank P. K. Mohanty for stimulating discussions and careful reading of the manuscript.
[1] M. E. Fisher, Am. J. Phys. 32, 343 (1964)

[2] G. S. Joyce, Phys. Rev. 155, 478 (1967)

[3] N. Bloembergen, Physica (Utrecht) 15, 386 (1949)

[4] L. van Hove, Phys. Rev. 95, 1374 (1954)

[5] D. Hone, C. Scherer, and F. Borsa, Phys. Rev. B 9, 965 (1974).

[6] F. Borsa and M. Mali, Phys. Rev. B 9, 2215 (1974).

[7] J-P. Boucher et al. , Phys. Rev. B 13, 4098 (1976).

[8] H. Benner, Phys. Rev. B 18, 319 (1978);

[9] A. Lagendijk and E. Siegel, Solid State Commun. 29, 709 (1976).

[10] A. V. Savin, G. P. Tsironis, and X. Zotos, Phys. Rev. B 72, 140402(R) (2005)

[11] D. Bagchi and P. K. Mohanty, arXiv:1206.2827 (to appear in Phys. Rev. B, 2012)

[12] H. Goldstein, C. P. Poole and J. L. Safko, Classical Mechanics, 3rd Edition, Addison Wesley.

[13] G. Müller, Phys. Rev. Lett. 60, 2787 (1988); 63, 813 (1989)
[14] R. W. Gerling and D. P. Landau, Phys. Rev. Lett. 63, 812 (1989); Phys. Rev. B 42, 8214 (1990).

[15] J. Liu, N. Srivastava, V. S. Viswanath, and G. Müller, J. Appl. Phys. 70, 6181 (1991)

[16] O. F. de Alcantara Bonfim and G. Reiter, Phys. Rev. Lett. 69, 367 (1992)

[17] M. Böhm, R. W. Gerling, and H. Leschke, Phys. Rev. Lett. 70, 248 (1993)

[18] S. W. Lovesey and E. Balcar, J. Phys.: Condens. Matter 6 (1994); S. W. Lovesey, E. Engdabl, A. Cuccoli, V. Tognetti and E. Balcar, J. Phys.: Condens. Matter 6 (1994)

[19] N. Srivastava, J. Liu, V. S. Viswanath, and G. Müller, J. Appl. Phys. 75, 6751 (1994)

[20] V. Constantoudis and N. Theodorakopoulos, Phys. Rev. E 55, 7612 (1997)

[21] S. Davis, and G. Gutiérrez, Physica B 355, 1 (2005)

[22] D. L. Huber, Physica B 407, 4274 (2012)

[23] R. Steinigeweg, EPL, 9767001 (2012) 